\title{
The Impact of COVID-19 on Mental Health: Community Development and Psychological Interventions During a Crisis
}

\author{
Matthew Cant* \\ University of Newcastle \\ Matthew_cant@aol.com
}

\begin{abstract}
COVID-19 has become a global pandemic which has caused disruptions in all walks of life. It has impacted the health of societies both physically, through infected individuals and the health-care systems trying to support them, and economically, as government interventions slow down business (especially those involving contact). Underappreciated, however, is how the current pandemic can impact mental-health, which is often poorly understood and more significantly, means that interventions designed to help individuals cope with distress are not always effective. The pandemic has caused significant psychological distress, and can often be compounded by government attempts to prevent transmission, which can be interpreted a loss of freedom, control and autonomy. Therefore, this paper has two main goals, which are to describe the psychological impact of COVID-19 on mental health (including what cognitive factors play a role in heightening distress) and what policies can be implemented by governments and health organizations to provide the infrastructure and required mental-health staff to aid those who are struggling with the pandemic.
\end{abstract}

Keywords: Covid-19, mental health, psychological intervention

\section{INTRODUCTION}

\section{COVID-19}

After just a number of months, the impact of the novel virus Coronavirus Disease-19 (COVID19)can be felt across the globe. Initially, an unidentified cluster of pneumonia in Wuhan, China, Covid-19 was quickly declared by WHO as a Public Health Emergency of International Concern [1], and has caused over 6 million confirmed cases, nearly 400,000 deaths in over 200 countries $\left(16^{\text {th }}\right.$ of June 2020). Information about the characteristics of Covid-19 infection is improving, with known symptoms including fever, cough, chills, breathing difficulties, sore throat, vomiting, nausea, myalgia, coughing, coryza and diarrhea. Furthermore, elderly men are more likely to contract the disease if they have medical comorbidities, and with more damaging outcomes; while severe reactions may also result in respiratory failure, cardiac injury, acute respiratory distress syndrome and death [2].

A provisional death rate was provided by WHO which stands at around $2 \%$, but the estimated range is somewhere between $0.3 \%$ and $0.6 \%$ [3]. Accordingly, the health care systems of many nations are struggling to cope with the influx of diseased patients and patient deaths, lead to many strict governmental interventions aimed at preventing transmissions, such as closing national borders, social-distancing, and complete lock-down and quarantine to restrict citizens mobility. However, the fear of the virus and the governmental interventions have disrupted economic stability, social functioning and people's lives, which has resulted in widespread psychological distress.

\section{COVID-19 and Mental Health}

In America, Congress has committed trillions of dollars in emergency funding for COVID-19, but only a fraction of that money was dedicated to mental-health., in a country where roughly 1 in 6 adults suffer from a mental illness under normal circumstances in a given year [4]. Unfortunately, America is characteristic of governments globally, and rates of mental illness are set to rise significantly across the world. For example, Wang et al., [5] recorded immediate psychological responses from 1210 participants from 194 cities in China, 3 weeks after the initial outbreak. They found that over half $(53.8 \%)$ of participants rated the psychological impact of the virus as moderate or severe, while moderate to severe symptoms were reported for depression (16.5\%), anxiety (28.8\%), and stress $(8.1 \%)$ respectively.

In Europe, Spain is one of the most severely impacted by COVID-19 and González-Sanguino et al., [6] investigated the consequences for mentalhealth for 3,480 Spanish residents. Similar to studies in China, a high degree of participants met the threshold for several psychological disorders. They found that $22 \%$ met the threshold for generalized anxiety disorder, 19\% for depression, and $16 \%$ for the post-traumatic-stress disorder (PTSD). It must be noted, however, that participants who met the thresholds for psychological disorders may not have disorders per say, it still highlights the distress caused by the virus nonetheless. 
Rajkumar [7] identified several studies investigating which groups were vulnerable to the psychological impact of COVID, and found that older adults, the homeless, migrant workers, the mentally ill, pregnant women, and Chinese students studying overseas were among those worst affected. Other studies have demonstrated that younger participants display higher symptoms of anxiety, depression and PTSD than older subjects, both during COVID-19 [6] and past epidemics like SARS [8]. Possibly, this reflects uncertainty for the future, as young people face suspended studies, talks of economic fallout and restricted social interactions. This reflects in suicides in Bangladesh, owing to the anticipated economic crash, and suicide has also been reported with individuals who believed they had been infected, though it was confirmed that they had not via autopsies $[9,10]$.

Brooks et al., [11] produced a rapid review of the psychological impact of quarantine and found that the combined loss of freedom, boredom and separation from loved ones can result in significant psychological distress. For example, responses such as depression, post-traumatic stress symptoms, emotional disturbance, irritability, low mood, and insomnia had a high prevalence in a sample who had potential contact with SARS either directly or indirectly. Although the literature is mixed as to what factors predispose people to distress during quarantine, the duration of quarantine is significantly associated with poorer mental-health, especially symptoms of post-traumatic stress, anger and avoidance behaviours. Likewise, anxiety and anger have been associated after release from quarantine up to 4-6 months by those with a history of psychiatric illness. Thus, understanding the psychological impact of novel virus outbreaks aids in carefully weighing the psychological costs of interventions like mass quarantine against its benefits [12], and where possible, informing attempts to limit the negative consequences associated with it.

\section{What is driving the psychological distress?}

Aside from the possible distress caused by government interventions like lock-down, and despite COVID-19 posing an objective threat, Paul Slovic highlighted that "risk does not exist independent of our minds and culture" (p.690) [13], and a significant degree of research supports the idea that the perception of risk is a subjective psychological phenomenon, influenced by cognitive, emotional, social, and individual variation, both between individuals and countries [14], [15].

Psychologists must consider how these factors function under the threat of a pandemic, and therefore, what cognitive mechanisms and traits predispose someone to respond more catastrophically to a crisis than others.
Understanding this, and how it relates to the broader context of COVID-19, can help to inform community interventions designed to reduce the emotional distress felt by the general public.

Cognitive appraisals are one such mechanism, a term coined by psychologist Robert Lazarus[16] to explain how we interpret the experiences that happen to us. Through this lens, we can understand how cognitive factors influence perceptions of risk and danger, and in turn, how this contributes to mental illness. When faced with significant environmental stress, we adopt various coping strategies to manage our physical and mental wellbeing. Cognitive appraisals are central to the coping process, which allow us to evaluate how a scenario is relevant to our well-being, and in what ways [17].

Cognitive appraisal associate with a range of emotional and behavioural outcomes in both immediate and long-term outcomes [17], and researchers have identified two main facets of appraisals, primary and secondary. Primary appraisals are the estimation of what one has at stake in a situation [17], while secondary appraisals refer to the perception of available actions to prevent harm or overcome the problem, which involves a complicated process of evaluating one's coping choices [17]. Little was known about COVID-19 when it first emerged and given the significant impact it has had, it was difficult for people to judge just how severe the virus was. As more and more countries went into lock-down, and the death toll kept increasing, this uncertainty could be extremely distressing for individuals.

Accordingly, the spread of information during a crisis is critical to how the public responses psychologically. In Wang et al.s' study in China, having access to up-to-date information was associated with significantly lower levels of depressive, anxious and stress-related symptoms, as well as higher engagement with precautionary behaviours (e.g. wearing a mask). Moreover, González-Sanguino et al. found that excessive information was associated with higher levels of psychopathological symptoms. Jonathan Haidt once said "the emotional tail wags the rational dog", highlighting how our cognitive appraisals are built on our emotional responses. Emotional responses precipitate and are crucial for, judgements of risk and decision making, otherwise known as an affect heuristic[18]. While a "healthy fear" is necessary to motivate precautionary behaviours; excessive or irrational fear can lead to detriments in mentalhealth. One of the determinants of risk perception is the ease and fluency with which something comes to mind.

Frightening thoughts and images occur to us with particular ease, and thoughts of danger that are more fluent and vivid also exacerbate fear. Given the mass global communication of accurate and 
misinformation through media outlets and social media, it's difficult not to be reminded of distressing nature of the pandemic. Estimates of causes, death rates and severity of a crisis are usually warped by media coverage, which is itself bias towards novelty and poignancy. As such, we do not see an exact replica of reality in our minds, but instead our expectations about the frequency of events are distorted by the prevalence and emotional intensity of messages to which we are exposed. In relation to primary and secondary appraisals, people who have been exposed to distorted information may estimate the virus through a catastrophic affect heuristic, while the loss of freedom due to governmental interventions and the nature of the virus can be interpreted as a loss of control and autonomy, thus sometimes compounding the distress.

Therefore it is the government's responsibility, as well as national and international health organizations to ensure the general public have access to accurate and up-to-date information to combat the spread of misinformation. It is also a necessity that psychological interventions are planned efficiently and effectively within a suitable infrastructure, and with a sufficient number of staff, to support the public, as well as those affected by COVID-19 directly, such as infected patients, families thereof, front-line health workers, and essential workers.

\section{Community Development and Intervention}

Any crisis interventions aimed at protecting communities from the impact of COVID-19 should deeply consider how to manage the psychological health of the individuals in those communities. Psychological interventions should be established on comprehensive assessments of risk factors which contribute to psychological distress, such as mental health status before a crisis, injury to self or family members, bereavement, life-threatening circumstances, separation from family, panic and household income [19]. One of the challenges of a pandemic is that community health services often do not have standardized training in clinical psychology or psychiatry, so may not know how to reduce the psychological distress of patients. Governments need to consider how to effectively provide psychological support when non-essential staff such as psychologists, clinical psychiatrists and mental health social workers could be put at risk during face-to-face interactions with patients.

Furthermore, although the number of mentalhealth practitioners (e.g. clinical psychologists, psychiatrists) is growing each year in different countries, these numbers are still insufficient to deal with the consequences of a global pandemic. For this reason, it is vital that governments focus resources into training mental health workers across different levels of society. Owing to these shortages, and the difficulty of exposing mentalhealth workers to the risk of infection, psychological interventions in certain communities may not always be feasible. Community intervention teams are sometimes comprised of volunteers, nurses, psychological counsellors, or teachers majoring in psychology and other relevant fields, but no members who are professionally trained or experienced in psychology or psychiatry [20]. This can lead to heavy caseloads and multiple responsibilities, which can decrease the effectiveness of psychological interventions.

The aforementioned issues can be mitigated by governmental policy to increase the national footprint of mental-health institutions. For example, improving relevant policies, optimizing organizational and managerial policies, strengthening personal training and constantly reviewing experiences in practice will increase the government's ability to efficiently mobilize resources and design effective interventions in response to public emergencies. Coinciding with government policy, it can ensure that essential health workers receive sufficient training in providing mental health support so that they can provide primary medical care and mental health care. Also, establishing professional teams of trained mental health practitioners specifically for crisis interventions in communities is essential[20], which help to effectively deal with the emotional distress and subsequent mental disorders caused by pandemics. A more recent development is the implementation of online mental health services, which have greatly improved the accessibility to mental health care.

In Brook et al.'s review, they identified a list of studies which have outlined specific therapeutic strategies for psychological interventions already in practice. For instance, establishing specialist teams qualified to manage emotional distress[20]; training in basic aspects of mental health care for community health personnel [20]; utilizing online surveys to examine mental health factors[21]; providing accurate and evidence-based education on mental health [22]; implementing self-help services and online counselling[21]; using structured letters as an asynchronous telepsychiatry consultation [23]; implementing synchronous telemedicine services for counselling as well as for diagnostic purposes [24]; and ensuring populations of a lower socioeconomic status can access online mental health services [25].

Interventions have been implemented with varying success, but represent a movement towards more comprehensive mental-health care and support for communities during times of crisis. They also have the advantage of improving access to mental health care with very little increase in infection of risk. The limitations of psychological interventions is that they depend on the availability 
of trained manpower and infrastructure, and it's not clear beforehand how they will be accepted by the general population. This is where government policies can work to build the required infrastructure, necessary manpower and educate the general population on both the challenges for mental health posed by a novel virus outbreak and what options are available to them. It's also clear that the interventions currently in use have not been thoroughly tested or validated in respective target populations. Thus mental-health institutions and psychological interventions should double as research opportunities, which will provide more accurate assessments of the issues and improving the effectiveness of the interventions themselves.

\section{CONCLUSION}

A variety of options are available to any government or institution aiming to improve their mental-health response capabilities, which will aid in dealing with the psychological problems caused by a pandemic like COVID-19. Broadly, based on the preceding literature, four main steps can be outlined, which consider actions relevant to the individual, the mental-health staff, and government policies enabling the mobilization of resources:

1) Understanding the psychological problem (Risk assessments; online surveys; online or telepsychiatry consultation)

2) Reviewing strengths and limitations of current community response capabilities (available resources; infrastructure and government policies; number of mental-health staff, including shortages)

3) Improving community response capabilities (reviewing and changing government policies; allocating resources to tackle mental-health problem; staff training in community mentalhealth care)

4) Designing and implementing effective psychological interventions (new and innovative methods, I.e. online mental-health care, online self-help services and counselling; education of the general population and mentalhealth staff; mental-health response teams specialized in public emergencies; reviewing and improving interventions and infrastructure ad hoc).

\section{REFERENCES}

[1] "Scientific American," 2020. [Online]. Available: Retrieved from https://www.scientificamerican.com/article /who-declares-coronavirus-outbreak-a global-health-emergency/.

[2] M. L. Holshue, C. DeBolt, S. Lindquist, K. H. Lofy, J. Wiesman, H. Bruce, ... and G. Diaz, "First case of 2019 novel coronavirus in the United States," New England Journal of Medicine., 2020.

[3] H. Nishiura, T. Kobayashi, Y. Yang, K. Hayashi, T. Miyama, R. Kinoshita, and A. R. Akhmetzhanov, "The rate of under ascertainment of novel coronavirus (2019$\mathrm{nCoV}$ ) infection: estimation using Japanese passengers data on evacuation flights," p. 419, 2020.

[4] National Institute of Mental Health, "Statistics," 2019. [Online]. Available: https://www.nimh.nih.gov/health/statistics/m ental-illness.shtml.

[5] C. Wang, R. Pan, X. Wan, Y. Tan, L. Xu, R. S. McIntyre and C. Ho, "A longitudinal study on the mental health of general population during the COVID-19 epidemic in China," Brain, behavior, and immunity, 2020.

[6] C. González-Sanguino, B. Ausín, M. ÁngelCastellanos, J. Saiz, A. López-Gómez, C. Ugidos and M. Muñoz, "Mental Health Consequences during the Initial Stage of the 2020 Coronavirus Pandemic (COVID-19) in Spain," Brain, Behavior, and Immunity., 2020.

[7] R. P. Rajkumar, "COVID-19 and mental health: A review of the existing literature," Asian journal of psychiatry, p. 102066, 2020.

[8] K. Sim, Y. H. Chan, P. N. Chong, H. C. Chua and S. W. Soon, "Psychosocial and coping responses within the community health care setting towards a national outbreak of an infectious disease," Journal of psychosomatic research, vol. 68, no. 2, pp. 195-202, 2010.

[9] K. Goyal, P. Chauhan, K. Chhikara, P. Gupta and M. P. Singh, "Fear of COVID 2019: First suicidal case in India!," Asian journal of psychiatry, vol. 49, p. 101989, 2020.

[10] A. I. Bhuiyan, N. Sakib, A. H. Pakpour, M. D. Griffiths and M. A. Mamun, "COVID19-related suicides in Bangladesh due to lock-down and economic factors: case study evidence from media reports," International Journal of Mental Health and Addiction, 2020.

[11] S. K. Brooks, R. K. Webster, L. E. Smith, L. Woodland, S. Wessely, N. Greenberg and G. J. Rubin, "The psychological impact of quarantine and how to reduce it: rapid review of the evidence," The Lancet, 2020.

[12] G. J. Rubin and S. Wessely, "The psychological effects of quarantining a city," Bmj, p. 368.

[13] P. Slovic, "Perception of Risk: Reflections on the Psychometric Paradigm," in Theories of Risk, New York, Praeger, 1992. 
[14] P. Slovic , B. Fischhoff and S. Lichtenstein, "Why study risk perception?," Risk analysis, vol. 2, no. 2, pp. 83-93, 1982.

[15] H. Joffe, "Risk: From perception to social representation," British journal of social psychology, vol. 42, no. 1, pp. 55-73, 2003.

[16] R. S. Lazarus, Psychological stress and the coping process, New York, NY: McGrawHill, 1966.

[17] S. Folkman, R. S. Lazarus, C. DunkelSchetter, A. DeLongis and R. J. Gruen, "Dynamics of a stressful encounter: cognitive appraisal, coping, and encounter outcomes," Journal of personality and social psychology, vol. 50, no. 5, p. 992, 1986.

[18] B. Fischhoff, P. Slovic, S. Lichtenstein, S. Read and B. Combs, "How safe is safe enough? A psychometric study of attitudes towards technological risks and benefits," Policy sciences, vol. 9, no. 2, pp. 127-152, 1978.

[19] P. Kun, S. Han, X. Chen and L. Yao, "Prevalence and risk factors for posttraumatic stress disorder: a cross-sectional study among survivors of the Wenchuan 2008 earthquake in China," Depression and Anxiety, vol. 26, no. 12, pp. 1134-1140, 2009.
[20] L. Duan and G. Zhu, "Psychological interventions for people affected by the COVID-19 epidemic," The Lancet Psychiatry, vol. 7, no. 4, pp. 300-302, 2020.

[21] Y. Liu, J. Li and Y. Feng, "Critical care response to a hospital outbreak of the 2019nCoV infection in Shenzhen, China.," 2020.

[22] S. Liu, L. Yang, C. Zhang, Y. T. Xiang, Z. Liu, S. Hu and B. Zhang, "Online mental health services in China during the COVID19 outbreak," The Lancet Psychiatry, vol. 7 , no. 4, pp. e17-e18, 2020.

[23] C. Xiao, "A novel approach of consultation on 2019 novel coronavirus (COVID-19)related psychological and mental problems: structured letter therapy," Psychiatry investigation, vol. 17, no. 2, p. 175, 2020.

[24] X. Zhou, C. L. Snoswell, L. E. Harding, M. Bambling and A. C. Smith, "The role of telehealth in reducing the mental health burden from COVID-19," Telemedicine and e-Health, vol. 26, no. 4, pp. 377-379, 2020.

[25] H. Yao, J. H. Chen and Y. F. Xu, "Rethinking online mental health services in China during the COVID-19 epidemic," Asian journal of psychiatry, vol. 50, p. 102015, 2020. 